\title{
Structural Studies of Multidrug Resistance Protein 1 Using "Almost" Cysless Template ${ }^{\mathbb{\Phi}}$
}

\author{
Daria N. Trofimova and Roger G. Deeley \\ Division of Cancer Biology and Genetics, Queen's Cancer Research Institute, Kingston, Ontario, Canada
}

Received September 20, 2017; accepted February 21, 2018

\begin{abstract}
Multidrug resistance protein, MRP1 (ABCC1) is a broad-spectrum ATP-binding cassette transporter that plays a major role in defense against dietary and environmental toxicants, in addition to contributing toward multidrug resistance of certain types of malignancy. Elucidating the molecular structure of hMRP1 is key to determining its mechanism of substrate recognition and transport. Here, we report the first successful attempt using cysteine-scanning mutagenesis coupled with cross-linking studies to probe the structure of hMRP1 in its native environment of the cell membrane or in membrane vesicles. We have established that an active 3 Cys $\triangle M R P 1$ (MRP ${ }_{204-1531}$ ) mutant, described in previous studies from our laboratory, is a suitable template with which to generate singleand double-cysteine mutants for performing cysteine mutagenesis studies. We have now used 3 Cys $\triangle M R P 1$ to probe the arrangement
\end{abstract}

of several TM segments, as well as the location of individual amino acids in these regions. Cysteine residues were introduced into TMs $8,14,15$, and 16 of 3 Cys $\triangle M R P 1$. The mutants were then subjected to chemical cross-linking analyses, and cross-linking was detected between the following cysteine pairs: Cys388 (TM7) and 11193C (TM16); Cys388 (TM7) and E1144C (TM15); R433C (TM8) and E1144C (TM15); and R433C (TM8) and T1082C (TM14). The aqueous accessibility of these residues and the possible implications of the differences between the open and closed states of the protein are also discussed. Moreover, using competition experiments involving a well characterized substrate and a cross-linking reagent for probing the Cys388/ I1193C mutant, we have defined these amino acid positions as a component of the potential site for estrone sulfate binding.

\section{Introduction}

Multidrug resistance protein MRP1 (ABCC1) belongs to the superfamily of ATP-binding cassette (ABC) transport proteins. MRP1 is a broad-specificity, transmembrane transporter that can efflux a vast range of amphiphilic organic anions, including many glutathione (GSH), glucuronate, and sulfate conjugates, as well as free GSH (Slot et al., 2011). It is ubiquitously expressed in the human body, with relatively high levels in the lung, testes, kidney, and blood organ barriers (Cole et al., 1992; Bakos and Homolya, 2007). The widespread tissue distribution of MRP1 implies an important physiologic role. However, an essential role in normal physiology remains to be firmly established. Studies in MRP1-null mice have revealed a number of intriguing potential functions, and MRP1 has been proposed to play a protective role in cells that express this protein (Leslie et al., 2005).

Clinically, MRP1 is the most extensively studied member of the multidrug resistance protein (MRP) subfamily of $\mathrm{ABC}$ transporters, particularly with respect to the development of multidrug resistancereversing agents. Its expression has been strongly linked to chemotherapy

This work was supported by the Canadian Institutes of Health Research [Grant MOP-97877].

https://doi.org/10.1124/dmd.117.078709.

S This article has supplemental material available at dmd.aspetjournals.org. response, time to relapse, and/or disease outcome in a number of tumor types, including breast, lung, prostate, and neuroblastoma (Chan et al., 1997; Hipfner et al., 1999).

The protein contains 17 transmembrane (TM) helices arranged in three polytopic transmembrane-spanning domains (MSDs), and two cytoplasmic nucleotide-binding domains (NBDs) (Cole, 2014). Biochemical and computational analyses indicate that TMs 1-5 constitute MSD0, and TMs 6-11 and TMs 12-17 constitute MSD1 and MSD2, respectively (Bakos et al., 1996; Hipfner et al., 1997).

Elucidating the arrangement of the TM segments, as well as the location of individual amino acids in them, is key to determining the mechanism of MRP1 substrate recognition and transport. TMs form the translocation pore of the protein and are also the site of substrate binding, as established through photoaffinity labeling and extensive mutagenesis studies (Deeley and Cole, 2006; Deeley et al., 2006). The drug-binding site for MRP1 has been proposed to be multipartite in nature, with substrates establishing different molecular contacts within the binding site (Deeley and Cole, 2006).

We have used cysteine mutagenesis, combined with cross-linking analysis, to investigate the relative position of residues within the hMRP1 TM helices that are predicted to be near the membrane/cytosol interface. Ideally, this strategy involves the introduction of two or more cysteine residues into an otherwise cysteineless (cysless) variant of a protein of interest. However, human MRP1 contains 25 cysteines and it

ABBREVIATIONS: aa, amino acid; ABC, ATP-binding cassette; AMP-PNP, adenylyl imidodiphosphate; cryo-EM, cryoelectron microscopy; CuPhen, copper phenanthroline; cysless, cysteineless; GSH, glutathione; HEK, human embryonic kidney; LTC 4 , leukotriene C4; M5M, 1,5-pentanediyl bismethanethiosulfonate; M8M, 3,6-dioxaoctane-1,8-diyl bismethanethiosulfonate; M17M, 3,6,9,12,15-pentaoxaheptadecane-1,17-diyl bismethanethiosulfonate; mAb, monoclonal antibody; MRP1, multidrug resistance protein 1; $\triangle \mathrm{MRP1}, \mathrm{MRP}_{204-1531}$; MSD, transmembrane-spanning domain; NBD, nucleotide-binding domain; PBS, phosphate-buffered saline; P-gp, P-glycoprotein; PM, plasma membrane; PNGase F, peptide: $N$-glycosidase F; TM, transmembrane. 
has not been possible to produce a cysless form of the protein that will traffic and function normally in animal cells. We have previously created an "almost cysless" $\triangle \mathrm{MRP1}$ (3Cys $\Delta \mathrm{MRP} 1$ ), which retains the functionalities of hMRP1 (Qin et al., 2012). This protein excludes MSD0 and contains cysteines at position 388 (TM7), as well as at positions 1439 and 1479, which are near the $\mathrm{COOH}$-terminal boundary of NBD2.

We first verified that $3 \mathrm{Cys} \triangle \mathrm{MRP} 1$ is a useful model for performing cysteine cross-linking studies, notwithstanding the presence of the three endogenous cysteine residues. Second, we introduced cysteine residues in the other TM helices and determined their ability to be cross-linked by bifunctional reagents, which allowed us to investigate the spatial relationships among several TM helices. Finally, we also used these cysteine residues as sensors to probe the aqueous accessibility of residues in the putative MRP1 translocation pore, and to investigate the localization of substrate binding sites by competition between substrates and cross-linking reagents.

We have compared these results obtained with the protein in its native membrane environment: with an extensively used computer-generated model of hMRP1, whose basis is the crystal structure of the bacterial halftransporter Staphylococcus aureus Sav1866, in the ADP-vanadatetrapped state, which is predicted to correspond to the low-affinity (closed) conformation of the protein (Dawson and Locher, 2006; DeGorter et al., 2008); and with the very recently published structures of bovine MRP1 (bMRP1) determined by cryoelectron microscopy (cryo-EM), in both apo- and substrate-bound forms (Johnson and Chen, 2017).

\section{Materials and Methods}

\section{Reagents}

1,5-Pentanediyl bismethanethiosulfonate (M5M), 3,6,9,12,15-pentaoxaheptadecane-1,17-diyl bismethanethiosulfonate (M17M), and 3,6-dioxaoctane-1,8-diyl bismethanethiosulfonate (M8M) were purchased from Toronto Research Chemicals Inc. (Toronto, ON, Canada). Rat monoclonal antibody $(\mathrm{mAb})$ MRPr1 was purchased from Enzo Life Sciences, Inc. (Lausen, Switzerland). Goat Anti-Rat Alexa Fluor 488, fluorescent dye Hoechst 33342, Lipofectamine 2000, Dulbecco's modified Eagle's medium, and fetal bovine serum were purchased from Invitrogen Canada Inc. (Burlington, ON, Canada). Mouse anti-His tag antibody, $\mathrm{E}_{2} 17 \beta \mathrm{G}$, estrone sulfate, ATP, and adenylyl imidodiphosphate (AMP-PNP) were all purchased from SigmaAldrich (St. Louis, MO). Horseradish peroxidase-labeled secondary antibody and G418 were purchased from Thermo Fisher Scientific (Mississauga, ON, Canada). Unlabeled leukotriene $\mathrm{C} 4\left(\mathrm{LTC}_{4}\right)$ was obtained from Calbiochem ( $\mathrm{La}$ Jolla, CA), and radiolabeled $\left[14,15,19,20-{ }^{3} \mathrm{H}\right] \mathrm{LTC}_{4}(172 \mathrm{Ci} / \mathrm{mmol})$ was purchased from Perkin Elmer Life Sciences (Woodbridge, ON, Canada). All primers used in this study were synthesized by Integrated DNA Technologies, Inc. (Coralville, IA).

\section{Construction of Mutants by Site-Directed Mutagenesis}

Mutations in TM8. Cysteine residues were introduced into 3Cys $\triangle M R P 1$ cDNA using QuikChange II Site-Directed Mutagenesis Kit (Stratagene California/Agilent Technologies, La Jolla, CA). The template used for mutagenesis was a pBlueScript II KS(+) vector containing a BamHI/Bsu36I fragment of human MRP1 cDNA [corresponding to 283-616 amino acids (aa)] containing C375I, C555A, and C563A mutations. A list of primers can be found in supplemental materials (Supplemental Table SI 1). After successful replacement and verification of the mutation by sequencing, which was performed at the Centre for Applied Genomics (Toronto, ON, Canada), the BamHI/Bsu36I fragment was cloned back into C-term 9xHis-tagged 3Cys $\triangle$ MRP1 cDNA in pcDNA 3.1(-) vector for the expression of mutant proteins in human embryonic kidney (HEK293) cells.

Mutations in TMs 14, 15, and 16. The pBlueScript II KS (+) vector with a Bsu36I/EcoRI fragment of human MRP1 cDNA (corresponding to 617-1294 aa), containing C682A, C730A, C744A, C984A, C1047A, C1105A, C1205A, and C1209A mutations, was used as the template for mutagenesis. A Bsu36I/EcoRI fragment was cloned into C-term 9xHis-tagged 3Cys $\triangle \mathrm{MRP} 1 \mathrm{cDNA}$ in a pcDNA 3.1(-) vector. In the case of mutants R433C/E1144C, R433C/S1145C, R433C/D1081C, and R433C/T1082C, a Bsu36I/EcoRI fragment was inserted into R433C 3Cys $\triangle$ MRP1 9xHis-tagged cDNA in a pcDNA 3.1(-) vector.

\section{Transfection, Selection, and Expression of MRP1 Protein}

HEK293 cells at $90 \%$ confluency $\left(1 \times 10^{6}\right.$ cells per well $)$ were transfected with $4 \mu \mathrm{g}$ of mutant cDNA using Lipofectamine 2000, according to the manufacturer's instructions. After 6 hours of incubation at $37^{\circ} \mathrm{C}$, transfection media was replaced by fresh Dulbecco's modified Eagle's medium supplemented with $7.5 \%$ fetal bovine serum. Four rounds of selection with $700 \mu \mathrm{g} / \mathrm{ml}$ G418 were performed. Cells were transferred and expanded on 150-mm diameter plates until confluency. Cells were then incubated at $28^{\circ} \mathrm{C}$ for another 24 hours in the presence of $50 \mathrm{mM}$ sodium butyrate and absence of G418. Confluent cells were pelleted, then washed in TB (Tris $50 \mathrm{mM}$, sucrose $250 \mathrm{mM}, \mathrm{pH}$ 7.4) supplemented with $1.25 \mathrm{mM} \mathrm{CaCl}_{2}$ and protease inhibitor cocktail (Hoffmann-La Roche Limited, Mississauga, ON, Canada) before being frozen at $-80^{\circ} \mathrm{C}$ until further use.

Plasma membrane vesicles of HEK293 were prepared as described previously (Loe et al., 1996). In brief, frozen pellets from HEK293 cells were disrupted by nitrogen cavitation, and plasma membrane vesicles were collected after repeated centrifugations and layering on a $35 \%(\mathrm{w} / \mathrm{v})$ sucrose cushion. Vesicles were then resuspended in TB through a 27.5-gauge needle, aliquoted, and stored at $-80^{\circ} \mathrm{C}$. Total protein concentration was determined by Bradford assay. Expression level of 3Cys $\triangle$ MRP1 proteins was determined by immunoblot analysis using an MRP1-specific mAb, MRP1r1 (Bakos et al., 1996). To distinguish between endogenously expressed full-length MRP1 and transiently expressed mutants, we used an anti-His tag antibody to probe the immunoblots.

\section{Plasma Membrane Trafficking and Glycosylation Status of Mutant Proteins}

Confocal microscopy was performed to verify the localization of mutants within cultured cells. In brief, HEK293 cells were seeded on poly-L-lysine-coated glass coverslips at $1 \times 10^{6}$ cells/well and transfected 24 hours later with Lipofectamine 2000 and $4 \mu \mathrm{g}$ of pcDNA3.1-containing mutants. After 6 hours of incubation at $37^{\circ} \mathrm{C}$, media was replaced with Dulbecco's modified Eagle's medium containing $50 \mathrm{mM}$ sodium butyrate and incubated at $28^{\circ} \mathrm{C}$ for a further 16 hours. Cells on coverslips were washed with phosphate-buffered saline (PBS), $\mathrm{pH} 7.4$, then fixed with $95 \%$ cold $\left(-70^{\circ} \mathrm{C}\right)$ ethanol for 10 minutes. 3Cys $\Delta \mathrm{MRP} 1$ proteins were detected using MRPr1 mAb as described above (Westlake et al., 2003), coupled with Alexa 488 fluorescent anti-rat secondary antibody. Nuclei were stained with Hoechst 33342 (1:5000). Cells were examined under a Quorum WaveFX-X1 spinning disk confocal microscope (Quorum Technologies Inc., Guelph, ON, Canada).

To assess the degree of glycosylation, all MRP1 mutants were subjected to peptide: $N$-glycosidase F (PNGase F) treatment. Membrane vesicles containing $10 \mu \mathrm{g}$ of total protein of 3Cys $\Delta \mathrm{MRP} 1$ mutants were treated with $1 \mu \mathrm{l}$ PNGase F (500 IU; New England Biolabs Ltd., Whitby, ON, Canada) according to manufacturer instructions. After treatment, protein samples were resolved by 7.5\% SDS-PAGE and probed with an anti-His tag antibody.

\section{Disulfide Cross-Linking Reaction}

Plasma membrane vesicles of mutants 3Cys $\Delta \mathrm{MRP1}$ (total protein: $10 \mu \mathrm{g}$ ) were resuspended in a total volume of $18 \mu \mathrm{l}$ hypotonic phosphate buffer $(10 \mathrm{mM}$ $\mathrm{Na}_{2} \mathrm{HPO}_{4}, 150 \mathrm{mM} \mathrm{NaCl}, \mathrm{pH}$ 7.2) to open up membrane vesicles and encourage plasma membranes to adopt a sheet-like conformation. The use of hypotonic buffer ensures that the membrane vesicles are burst open and, thus, that the MRP1 protein is accessible from both sides of the membrane (Rothnie et al., 2006). This allowed thiosulfonate cross-linking reagents to access cysteines located on both sides of the cell membrane. The plasma membrane sheet preparation was treated with $2 \mu \mathrm{l}$ of cross-linking reagent (final concentration $0.5 \mathrm{mM}$ ) for 15 minutes on ice. The reaction was stopped by addition of EDTA solution to a final concentration of $80 \mathrm{mM}$, followed by the addition of $2 \times$ SDS sample loading buffer [Tris $0.5 \mathrm{M}, \mathrm{pH} 6.8,20 \%$ (v/v) glycerol, $4.4 \%$ (w/v) SDS, and no reducing agent]. Reaction mixtures were then loaded and run on a 7.5\% SDS-PAGE gel. Western blotting was used to analyze protein samples using mouse anti-His tag antibody, which recognizes His-tagged mutants of MRP1. 
The disulfide cross-linking reaction for each mutant was performed at least two times. For each protein mutant two independent expressions and membrane vesicle preparations were obtained.

\section{Competition of Substrate with Cross-Linker}

For the competition experiments, plasma membranes of 3Cys $\Delta \mathrm{MRP} 1$ mutants were first incubated with various concentrations of either $\mathrm{LTC}_{4}(0-1000 \mathrm{nM})$ or estrone sulfate $(0-75 \mu \mathrm{M})$, in presence of $10 \mathrm{mM} \mathrm{MgCl}_{2}$, for 15 minutes at $22^{\circ} \mathrm{C}$. In the case of estrone sulfate, $1 \mathrm{mM} S$-methyl-GSH was also added. Reaction mixtures were cooled on ice for 5 minutes and cross-linked under the conditions described above. Samples were then analyzed by immunoblotting with anti-His tag primary antibody.

\section{Aqueous Medium Accessibility Test}

For labeling of whole cells, cells transfected with cDNA of a mutant from two 150 -mm confluent plates were harvested by centrifugation at $230 \mathrm{~g}$ for 10 minutes at $4{ }^{\circ} \mathrm{C}$. Cell pellets were washed twice with $10 \mathrm{ml}$ of cold PBS, then resuspended with $5 \mathrm{ml}$ of $2.5 \mathrm{mM}$ 2-aminoethyl methanesulfonate (MTSEA), $5 \mathrm{ml}$ of $10 \mathrm{mM}$ sodium 2-sulfonatoethylmethane thiosulfonate (MTSES), or $5 \mathrm{ml}$ of PBS solution, and incubated at room temperature for 15 minutes. Different concentrations of these two compounds were used owing to their specific reactivity with cysteine (Stauffer and Karlin, 1994). The cells were then washed three times with $10 \mathrm{ml}$ of cold PBS to remove unreacted chemicals. Cell pellets were frozen and kept at $-80^{\circ} \mathrm{C}$ before being used to prepare plasma membrane vesicles according to the protocol described above.

To test the effect of MTSEA and MTSES on cross-linking, the plasma membranes (10 $\mu \mathrm{g}$ of total protein) were resuspended in hypotonic phosphate buffer and the membrane sheets obtained were treated with $50 \mu \mathrm{M}$ thiosulfonate cross-linker (M5M or M8M) for 15 minutes on ice. The reactions were stopped by addition of a solution of EDTA containing $2 \times$ SDS sample loading buffer, as described above, and analyzed by immunoblot assay.

For labeling of membrane vesicles, untreated mutant cells were harvested by centrifugation at $230 \mathrm{~g}$ for 10 minutes at $4^{\circ} \mathrm{C}$. Cell pellets were frozen and kept at $-80^{\circ} \mathrm{C}$. Plasma membrane vesicles were obtained according to the protocol described above. Plasma membranes ( $10 \mu \mathrm{g}$ of total proteins) were resuspended in TB and treated with $2.5 \mathrm{mM}$ MTSEA, $10 \mathrm{mM}$ sodium MTSES, or TB for 15 minutes at room temperature. MTSEA or MTSES reagents were removed from membrane vesicles using G-50 spin columns equilibrated with TB. Flow-through fractions rich in membrane vesicles were then treated with $50 \mu \mathrm{M}$ M5M or M8M, or without a cross-linker, for 15 minutes on ice. The reactions were stopped with $2 \times$ SDS sample loading buffer containing EDTA and no reducing agent, and then run on $7.5 \%$ SDS-PAGE gel, followed by immunoblot analysis with anti-His tag antibody.

\section{ATP-Dependent Uptake of ${ }^{3} \mathrm{H}$-Labeled $\mathrm{LTC}_{4}$ by $3 \mathrm{Cys} \Delta \mathrm{MRP1}$ and Its Mutants}

Vesicular transport activity of 3 Cys $\triangle \mathrm{MRP1}$ mutants was measured using plasma membrane vesicles prepared from transfected HEK293 cells. A one-timepoint $\left[{ }^{3} \mathrm{H}\right] \mathrm{LTC}_{4}\left(50 \mathrm{nM}, 20 \mathrm{nCi} /\right.$ reaction) uptake assay was performed at $25^{\circ} \mathrm{C}$ for 3 minutes in 50- $\mu 1$ reaction volume containing $4 \mathrm{mM}$ ATP (or $4 \mathrm{mM}$ AMP-PNP in the control group), $10 \mathrm{mM} \mathrm{MgCl}_{2}$, and membrane vesicles containing $0.1-0.5 \mu \mathrm{g}$ of MRP1 (estimated by immunoblotting analysis). The reactions were stopped after 3 minutes by rapid dilution with $1 \mathrm{ml}$ of ice-cold TB buffer $(\mathrm{pH} 7.4)$ and filtrated through glass fiber filters (type A/E, pore size $1 \mu \mathrm{m}$; Pall Life Sciences, Ann Arbor, MI). Filters were washed twice with $4 \mathrm{ml}$ of TB and remaining radioactivity was measured by liquid scintillation counting. ATP-dependent drug uptake was determined by subtracting the uptake of $\mathrm{LTC}_{4}$ in the presence of AMP-PNP from the uptake in the presence of ATP. All transport assays were performed in triplicate, with the results presented as mean \pm S.D.

\section{Results}

3Cys $\triangle$ MRP1 Is a Suitable Template for Cysteine Mutagenesis and Cross-Linking Studies. We have previously described functional studies of the 3Cys $\triangle \mathrm{MRP} 1 \mathrm{C}$-term 9xHis tag construct that was used for the cysteine mutagenesis (Qin et al., 2012). 3Cys $\Delta \mathrm{MRP} 1$ is a truncated form of MRP1 lacking MSD0 (aa 1-203), with all remaining cysteines replaced with either Ala, Ser, or Ile, except three endogenous cysteines at positions 388,1439 , and 1479 . These remaining cysteines, located in TM7 (Cys388) and NBD2 (Cys1439 and Cys1479), are depicted in Fig. 1. According to the existing model of hMRP1, the distances between Cys388 and Cys1439 and between Cys388 and Cys1479 (51 and $66 \AA$, respectively), could not be cross-linked by the bifunctional thiosulfonate reagent M17M, which has a spacer arm of $23 \AA$. On the other hand, the predicted distance between Cys1439 and Cys1479 is $17 \AA$, which hypothetically could be cross-linked by the bifunctional thiosulfonate reagent M17M. To test this hypothesis, cross-linking reactions of 3Cys $\triangle \mathrm{MRP} 19 \mathrm{xHis}$ tag were performed with three bifunctional thiosulfonate reagents of various spacer lengths.

These reagents cross-link cysteines depending on the distances between their $\alpha$-carbon atoms. The bifunctional thiosulfonate reagents used in the present study-M5M, M8M, and M17M-cross-link cysteines where their $\alpha$-carbons are separated by approximately 9,13 , and $23 \AA$, respectively. If a cross-linking reaction takes place, it can be detected by a shift in electrophoretic mobility on SDS-PAGE toward a larger molecular-weight species (Loo and Clarke, 1996). All three thiosulfonate reagents failed to induce intramolecular cross-linking of any of the three endogenous cysteines found in 3Cys $\Delta \mathrm{MRP} 1$ (data not shown). However, products with apparent molecular weights in the range of $350 \mathrm{kDa}$ and higher were observed, which were presumed to represent intermolecular cross-linked products of 3Cys $\Delta \mathrm{MRP} 1$. These results suggested that 3 Cys $\triangle \mathrm{MRP} 1$ was a suitable template for performing cysteine mutagenesis studies.

Cross-Linking Studies of Residues Predicted to Be Close to the Membrane/Cytosol Interface. One of the essential endogenous cysteines, Cys388, is predicted to be close to the membrane/cytosol interface in TM7, on the basis of the hMRP1 Sav1866 model, with its side chain possibly oriented toward TM16 (DeGorter et al., 2008). Thus, we began our studies by examining the ability of Cys388 to become cross-linked to Cys residues introduced into TM16 (Fig. 2A). We chose to mutate the I1193 and Y1190 residues, as their side chains are predicted to point toward TM7; their distances from Cys388 are approximately 6 and $6.7 \AA$, respectively. Notably, Y1190 has been shown to be important for transport activity of MRP1. The substitution of Y1190 with Ala, Ser, or Val reduced the transport of several substrates (Conseil et al., 2005) but did not significantly alter the expression of MRP1. On the basis of these data we predicted that these sites would be good locations for the introduction of cysteines.

cDNAs of all mutants described in this study were transfected into HEK293 cells, grown until confluency, and then harvested, frozen, and later used to obtain membrane vesicles. Confocal microscopy showed correct mutant trafficking, i.e., the proteins resided mostly in the plasma membrane (PM). A confocal microscopy image of mutant I1 193C 3Cys $\triangle \mathrm{MRP} 1$ is provided in the supplementary materials section (Supplemental Fig. SI 1). Immunoblotting experiments indicated that I1193C and Y1190C mutants were successfully expressed. The level of mutant expression was approximately $50 \%$ lower than that of 3Cys $\Delta \mathrm{MRP} 1$. To assess the degree of glycosylation, both mutant proteins were subjected to PNGase F treatment, which showed the presence of fully glycosylated proteins (Supplemental Fig. SI 2).

Aliquots of membrane vesicles containing $10 \mu \mathrm{g}$ of total protein were resuspended in phosphate buffer and treated with thiol cross-linking reagents [M5M, M8M, M17M, and copper phenanthroline (CuPhen)] for 15 minutes on ice. Immunoblot analysis of mutants separated by SDS-PAGE revealed that a cross-linking reaction occurred in mutant I1193C 3Cys $\Delta$ MRP1, between Cys388 and Cys1193 (Fig. 3A), but not in mutant Y1190C 3Cys $\Delta$ MRP1 (data not shown). The band of crosslinked protein (X-link) had an apparent molecular mass of $220 \mathrm{kDa}$ and 

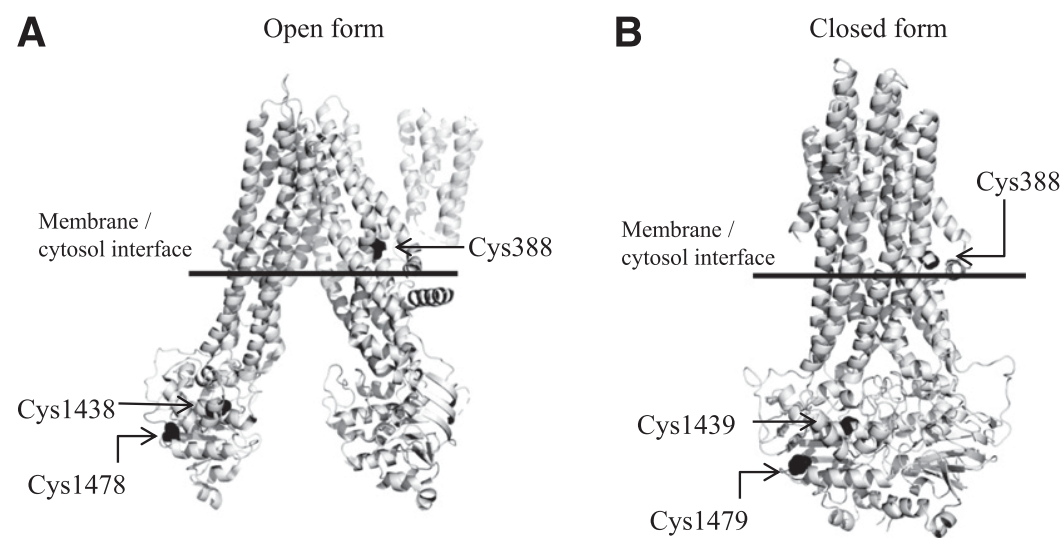

Fig. 1. Positions of endogenous Cys388, Cys1439, and Cys1497 (represented by black spheres) in (A) cryo-EM structure bMRP1 (205-1516 aa, PDB ID: 5uj9) and (B) Sav1866-based hMRP1 model (300-1531 aa). The sequence of hMRP1 is $91 \%$ identical to bMRP1. According to the sequence amino acid, Cys, in human and bovine proteins located at the position 388, however Cys 1439 (hMRP1) is located at position 1438 in bMRP1 protein, as well as Cys1479 is at 1478, respectively. Cys388 is situated in TM7; Cys1439 and Cys1497 are located in NBD2. Images were created using PyMOL software (Delano Scientific LLC, San Carlos, CA) The hMRP1 model was aligned to the bMRP1 structure using align tool in PyMOL automatically. (A) and (B) show protein molecule from the same view point.

was lost in the presence of reducing agent, suggesting the presence of an internal thiosulfonate bridge, as expected (data not shown).

The three thiosulfonate cross-linking reagents-M5M (9 ̊), M8M (13 $)$ ), and M17M (23 A) —all generated a cross-linked product with the I1193C mutant. In contrast, CuPhen, the shortest of the cross-linkers (5 A), failed to do so. These results suggested that TMs 7 and 16 are situated adjacent to each other at the membrane/cytosol interface, greater than $5 \AA$ apart, but less than $9 \AA$. This finding is in agreement with the Sav1866 model of hMRP1, where TMs 7 and 16 helices are located close together, with a predicted distance of $6 \AA$ between Cys 388 and I1193. However, in recently published cryo-EM molecular structure of bMRP1 (Johnson and Chen, 2017) which is depicted in its apo state, Cys 388 points toward TM8 rather than TM16 (Fig. 2B). In such an orientation cross-linking of Cys388 to I1193 (TM16) would not be expected. The crystal structure of another ABC transporter, mouse P-glycoprotein (P-gp), is also available (Aller et al., 2009). The structure was obtained in an open conformation. Cys133 in mP-gp occupies a comparable position to Cys388 in hMRP1 (Fig. 2C) and in the crystal structure projects into the translocation pore of P-gp toward TM16. This orientation of Cys 133 in P-gp correlates well with our observation.

To determine the optimum cross-linking conditions, we investigated the effects of different cross-linker concentrations and reaction times. Experiments were carried out at $4{ }^{\circ} \mathrm{C}$ to reduce thermal motion of the protein. Cross-linking of the mutant I1193C occurred rapidly, with the majority of the cross-linked product detected within 2 minutes (Fig. 4B).

Plasma membrane vesicles prepared from cells expressing the I1193C mutant were treated with various concentrations of M5M cross-linker $(0-50 \mu \mathrm{M})$ for 15 minutes. The majority of the observed cross-linking occurred with M5M concentrations of 5-50 $\mu \mathrm{M}$ M5M (Fig. 4A). With increasing M5M concentrations, the amount of intramolecular species (X-link) increased, whereas levels of intermolecular species (350$400 \mathrm{kDa}$ ) remained largely unchanged. The protein band attributed to non-cross-linked protein $(150 \mathrm{kDa})$ disappeared in reactions with 5-50 $\mu \mathrm{M}$ cross-linker, confirming that the vast majority of the protein was modified by the cross-linker.

According to the hMRP1 model, TMs 7 and 15 are situated on opposing sides of the translocation pore, yet remain relatively close to each other (Fig. 2A). To investigate the location of TM15 relative to TM7, we selected two amino acids, E1144 and S1145, which are believed to project into the translocation pore and to lie close to Cys388 in TM7. On the basis of the hMRP1 model, the estimated distance of Cys388 (TM7) from E1144 and S1145 (TM15) was 13.8 and $18.6 \AA$, respectively. Conseil et al. (2009) substituted E1144 with Ala and found that although the E1144A mutant was well expressed, the transport of estrone sulfate and $\mathrm{E}_{2} 17 \beta \mathrm{G}$ was reduced.

We produced two mutants of 3Cys $\Delta$ MRP1: E1144C and S1145C. Plasma membrane sheets of the mutants were subjected to cross-linking reactions with each of the four thiol cross-linking reagents. Samples were analyzed by SDS-PAGE, followed by immunoblot detection. As shown in Fig. 3B, the longest reagent M17M (23 A) —but not CuPhen, M5M, or M8M-cross-linked Cys1144 (TM15) to Cys388 (TM7). In contrast, Cys1145 failed to cross-link to Cys388 (TM7) with any length of cross-linker (data not shown). Despite the prediction that the distance between these cysteines is short enough to be cross-linked by M17M, the alignment of the side chains may not permit such cross-linking to occur. Nevertheless, given that cross-linking occurred between Cys388 and E1144C, we can conclude that TMs 7 and 15 comprise regions of the translocation pore, with an approximate distance between them of less than $23 \AA$, which is in reasonable agreement with the hMRP1 model. The fact that E1144C (TM15) was strongly cross-linked suggests that in the open conformation, Cys388 (TM7) may be rotated more toward the interior of the translocation pore than the model hMRP1 in the closed conformation predicts. This is consistent with the observation that the comparable residue in P-gp (Fig. 2C) projects into the translocation pore (open conformation). It is interesting to note that both bMRP1 structures (Fig. 2B) depict Cys388 faced toward TM8, but not TM15. Contrary to

TABLE 1

Summary of cross-linking reaction of single and double mutants of $3 C y s \Delta M R P 1$

Numbers correspond to the thiosulfonate reagent that cross-linked cysteine residues in a given mutant. For instance, "5" corresponds to M5M. Dash (-) indicates no cross-linked product detected with any of the three cross-linkers. Empty cells indicate that the cross-linking reactions were not performed.

\begin{tabular}{|c|c|c|c|c|c|c|c|}
\hline \multirow{2}{*}{ Mutant } & \multirow{2}{*}{$\frac{\mathrm{TM} 8}{\mathrm{R} 433 \mathrm{C}}$} & \multicolumn{2}{|c|}{ TM16 } & \multicolumn{2}{|c|}{ TM15 } & \multicolumn{2}{|c|}{ TM14 } \\
\hline & & Y1190C & I1 193C & E1144C & S1145C & D1081C & $\mathrm{T} 1082 \mathrm{C}$ \\
\hline 3Cys $\Delta \mathrm{MRP} 1$ & - & - & $5,8,17$ & 17 & - & - & - \\
\hline R433C 3Cys $\Delta$ MRP1 & & & & $5,8,17$ & 8,17 & - & 17 \\
\hline
\end{tabular}


A

\section{hMRP1 model}

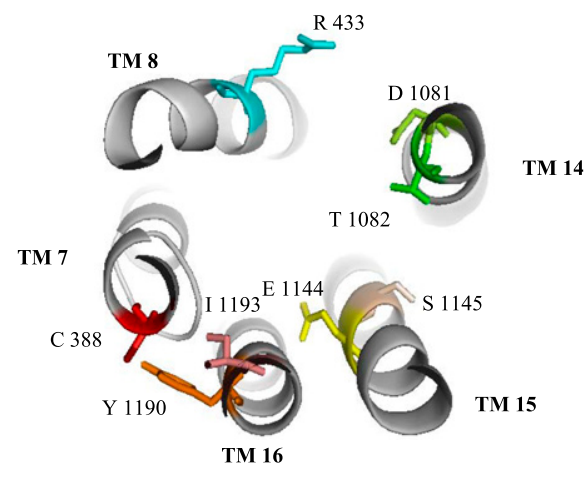

B bMRP1 cryo-EM

C mP-gp crystal structure
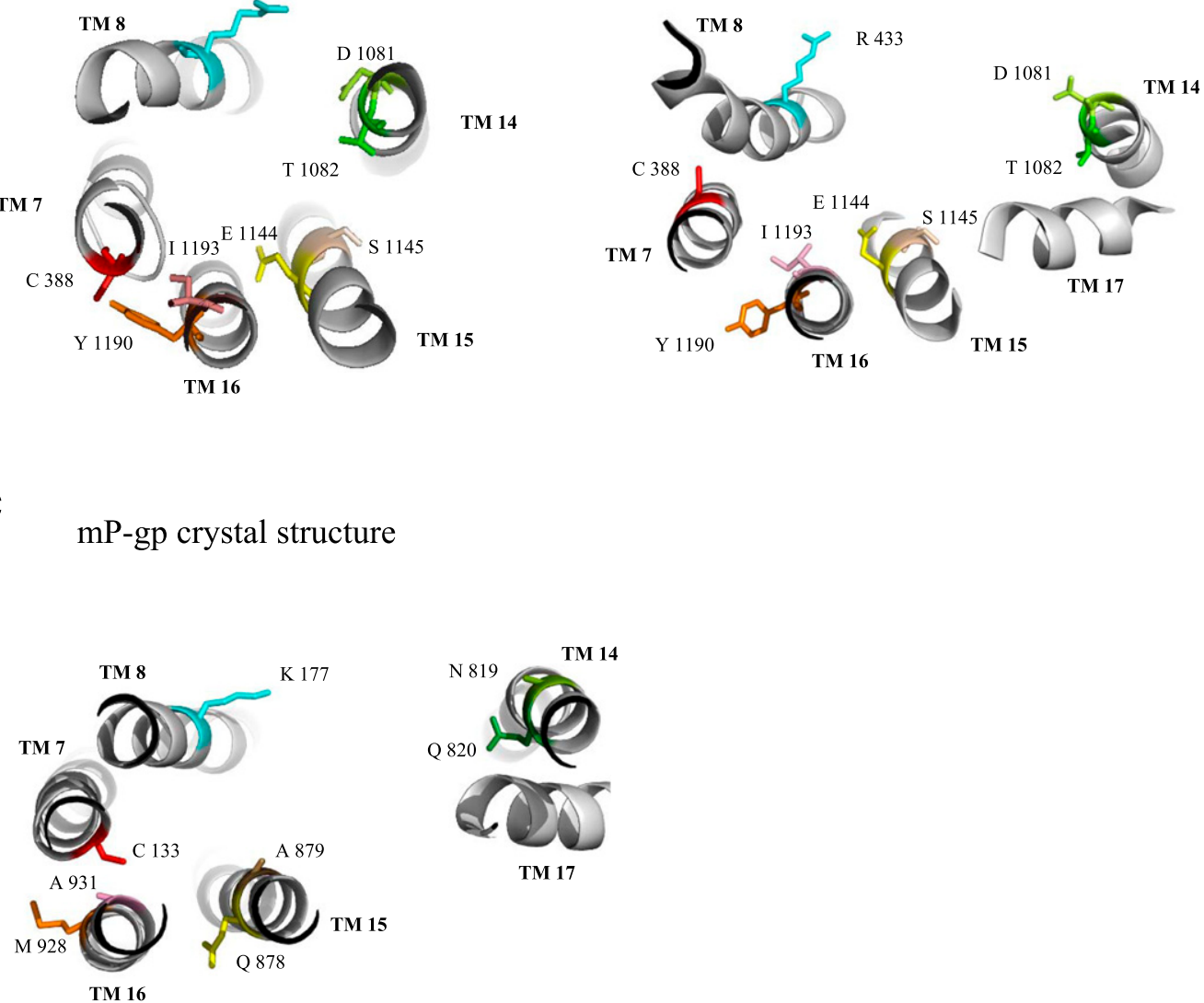

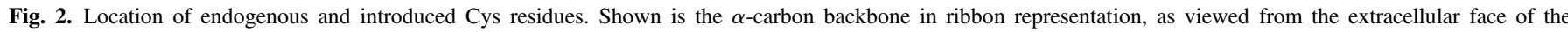

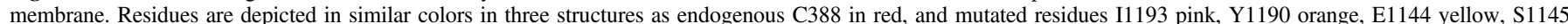

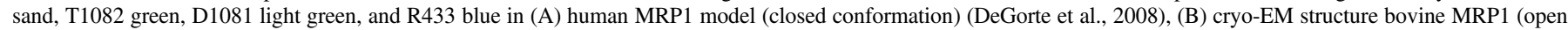

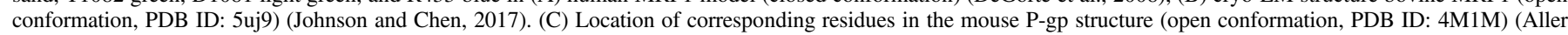
et al., 2009).

our results, such an orientation of Cys 388 and E1144C would appear to be unfavorable for cross-linking.

Cross-linking analyses of I1193C (TM16) and E1144C (TM15) mutants strongly suggest that in the open conformation, Cys388 (TM7) rotates more toward the interior of the translocation pore. Therefore, we explored the possibility of cross-linking Cys388 (TM7) with selected amino acid residues in TM14.

TM14 is predicted to be approximately $21 \AA$ from Cys388 (TM7) (hMRP1 model) and thus could potentially be cross-linked by the longest reagent, M17M (23 $\AA$ ). We investigated this possibility by introducing cysteines at positions 1081 and 1082 in TM14. According to the hMRP1 model, residues at these positions face both the translocation pore and TM7. The predicted distances between C388 and D1081 and between C388 and T1082 are 23.6 and 20.5 A, respectively. Moreover, we have shown previously that mutation of the residue at position T1082A does not alter the expression or the transport activity of MRP1 (Zhang et al., 2003).

Membrane vesicles from the 3Cys $\Delta$ MRP1 D1081C and T1082C mutants were probed with three cross-linking thiosulfonate reagents of differing lengths. Immunoblot analysis revealed no shift in the mobility of mutants, indicating that the two mutants were not cross-linked. The absence of cross-linking in these mutants could be attributed, in part, to differences between the open and closed conformations of hMRP1.
Although the current model represents hMRP1 in an ADP-trapped (closed) conformation, MRP1 in isolated membrane vesicles most likely exists in an open conformation. In the latter conformation, TMs 7 and 14 may be situated further apart (30-35 $\AA$ ), as seen in both the P-gp structure (Aller et al., 2009) and bMRP1 (Johnson and Chen, 2017), which would prevent their cross-linking by even the longest reagent, M17M (23 ̊). Taguchi et al. (1997) reported that the addition of ATP in the presence of vanadate traps MRP1 in a closed state; thus, one would expect cross-linking to occur in the vanadate-trapped state (on the basis of the hMRP1 model). We tested this premise by performing crosslinking experiments in the presence of ATP and vanadate. No crosslinked proteins were formed under these conditions (data not shown). These results therefore failed to provide evidence that TMs 7 and 14 are situated 20.5-24 A from each other, as predicted by the hMRP1 model (closed conformation). It is interesting to note that in the recently reported $\mathrm{LTC}_{4}$-bound structure of bMRP1 (closed conformation), residues Cys 388 (TM7) and D1081C or Cys 388 (TM7) and T1082C (TM14) were predicted to be 30 or $27 \AA$ apart and thus should not be cross-linked even by the longest cross-linking reagent used in this study, which is consistent with our findings.

The mutations of TM15 (E1144C, S1145C) and TM14 (D1081C, T1082C) described above targeted residues predicted to project into the translocation pore of MRP1. The opposite side of the translocation pore 
A

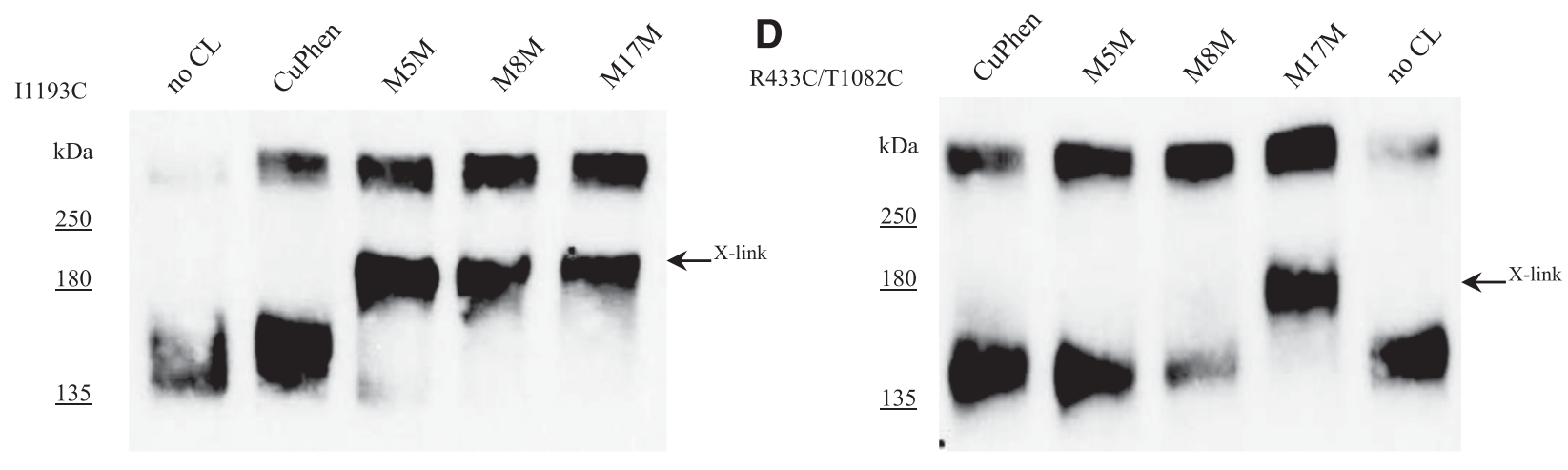

B
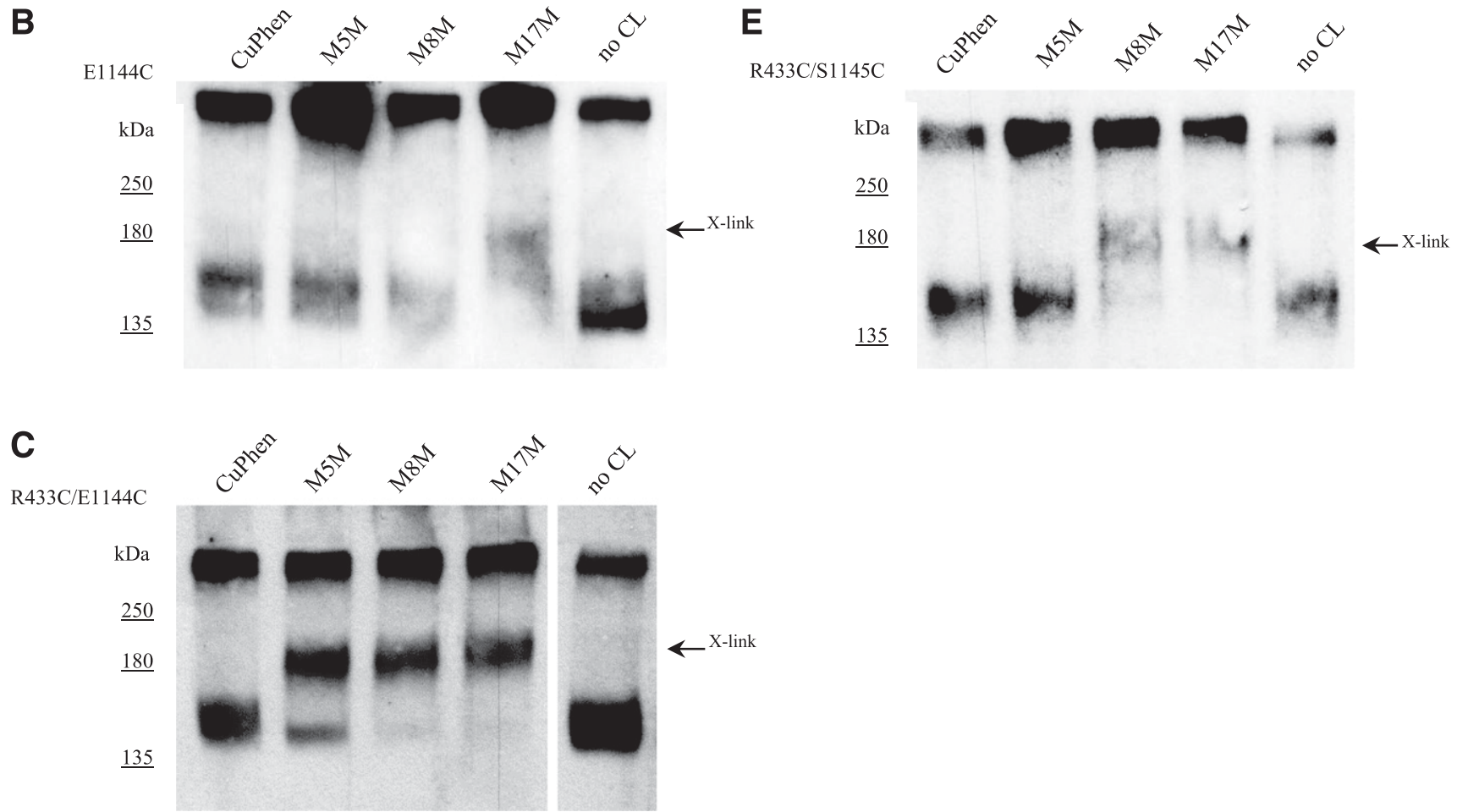

Fig. 3. Cross-linking of 3Cys $\triangle \mathrm{MRP} 1$ mutants containing pairs of cysteines. Membranes were prepared from HEK293 cells that expressed mutants I1193C (A), E1144C (B), R433C/E1144C (C), R433C/T1082C (D), and R433C/S1145C (E) of 3Cys $\Delta$ MRP1. Membrane sheets were treated without (no cross-linking) and with cross-linker (CuPhen, M5M, M8M, M17M) for 15 minutes on ice. Final concentration of cross-linking reagents in the reactions was $50 \mu$ M. Reactions were stopped by addition of SDS loading buffer containing EDTA and no thiol-reducing agent, and samples were subsequently subjected to immunoblot analysis. Membranes were probed with anti-His tag antibody. Representative immunoblot from at least two independent cross-linking experiments is shown. For each protein mutant two independent protein expressions and membrane vesicle preparations were obtained. The position of the cross-linked product is indicated (X-link).

is predicted to include TM8, with the ionic side chains of residues $\mathrm{R} 433$ and Q432 projecting into the pore (Fig. 2A). We chose to introduce a cysteine at position 433 , given that $\mathrm{R} 433$ is the site of a naturally occurring Ser polymorphism that results in decreased $\mathrm{LTC}_{4}$ and estrone sulfate transport, as well as increased doxorubicin resistance (Conrad et al., 2002). The level of expression of the R433C mutant remained unaltered. Consequently, we generated double mutants with R433C (TM8), together with one of the previously introduced cysteines in TMs 14 or 15 , to investigate the position of TM8 relative to TMs 14 and 15 . We created two mutants (R433C/D1081C and R433C/T1082C) with cysteine substitutions in both TMs 8 and 14. Two other double mutants (R433C/E1144C and R433C/S1145C) were also generated, in which we replaced cysteines in TMs 8 and 15. Plasma membrane vesicles from these mutants were prepared and exposed to various cross-linkers. The mutant of TMs 8 and 15 (R433C/E1144C) was efficiently cross-linked by all three thiosulfonate reagents: M5M (9 ̊), M8M (13 ̊̊), and M17M
(23 ^) (Fig. 3C). The R433C/S1145C mutant was cross-linked by thiosulfonate reagents M8M (13 ̊̊) and M17M (23 A), but not by M5M or CuPhen (Fig. 3E). The cross-linking ability of these mutants confirms the close proximity between TMs 8 and 15 (approximately $9 \AA$ ).

According to the hMRP1 model (closed conformation), the predicted distances between R433C (TM8) and E1144C (TM15) and between R433C and S1145C (TM15) are 16.1 and $17.1 \AA$, respectively. In the bMRP1 structure (open conformation), the distances between the same pairs are 17.1 and $17.4 \AA$ (18.0 and $14.6 \AA$ in closed conformation). By comparison, in the P-gp crystal structure, these distances are 17.9 and $13.8 \AA$ for both closed and open forms of the protein. However, our experiments revealed that these distances are much shorter than reported in all three structures. This might be explained if R433 is actually rotated more toward the translocation pore.

Cross-linking analysis of TMs 8 and 14 double mutants showed that R433C/T1082C can be cross-linked by M17M (23 Å; Fig. 3D), whereas, 
A

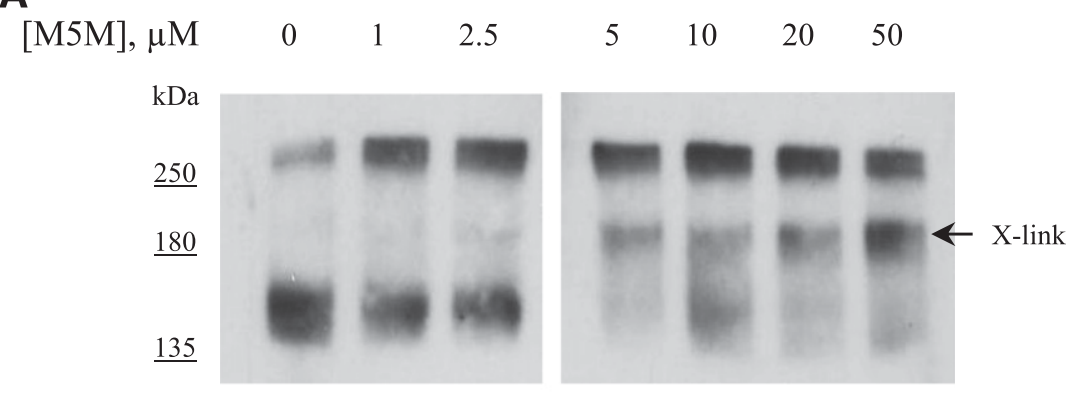

B

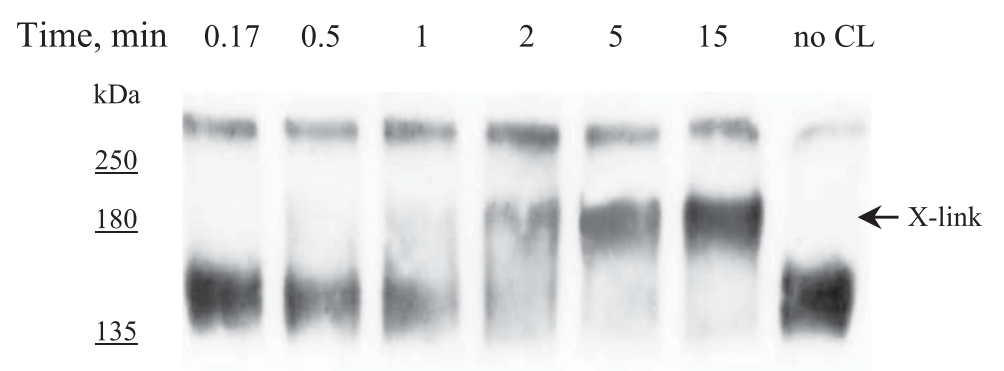

Fig. 4. Effect of M5M concentration and reaction time on crosslinking of I1193C 3Cys $\Delta$ MRP1. Membranes were prepared from HEK293 cells transiently expressing the I1193C mutant of 3Cys $\triangle \mathrm{MRP} 1$. The membranes sheets were incubated with various concentrations $(0-50 \mu \mathrm{M})$ of M5M for 15 minutes on ice (A), or with $50 \mu \mathrm{M}$ M5M for the indicated times (minutes) (B). The reactions were stopped by addition SDS loading buffer containing EDTA but no reducing agent. Samples were then subjected to immunoblot analysis. The position of cross-linked protein is indicated (X-link).

R433C/D1081C showed only barely detectable cross-linking with M17M (data not shown). Neither mutant was cross-linked with any of the shorter cross-linkers, such as CuPhen, M5M, and M8M. Crosslinking analysis of R433C/D1081C and R433C/T1082C suggested that D1081 and T1082 are located quite distantly from R433 despite the fact that TM8 and TM14 are predicted to be adjacent to each other. According to the hMRP1 model the distance between TM8 and TM14, in particular R433 and D1081, is $11.2 \AA$; thus, we expected to see cross-linking by M8M (13 $\AA$ ) but did not. This discrepancy could be attributed to the differences in conformation of the protein between the model, which represents the protein in a closed form (ADP-trapped), whereas in membrane vesicles, in the absence of a nucleotide and a substrate, the protein is expected to be in an open conformation. The bMRP1 structure (apo form) should likewise represent the protein in the open conformation. However, the distance of the R433C/D1081C pair and $\mathrm{R} 433 \mathrm{C} / \mathrm{T} 1082 \mathrm{C}$ in bMRP1 is 25.8 and $26.9 \AA$, respectively, and should not be cross-linked even by the longest M17M cross-linker (23 ̊). This is inconsistent with our findings, as cross-linking was observed for the R433C/T1082C mutant. Our experimentally obtained distance (more than 13 but less than $23 \AA$ ) is similar to bMRP1 (in the presence of $\mathrm{LTC}_{4}$ ), where R433/D1081 and R433/T1082 are situated at a distance of 18.3 and $20.5 \AA$, accordingly.

Given that R433C (TM8) can be cross-linked to E1144C (TM15), and that Cys388 (TM7) cross-links with I1193C (TM16) and E1144C (TM15), we examined whether R433C (TM8) can cross-link with Cys388 (TM7). Immunoblot analysis of plasma membranes prepared from cells transfected with the R433C mutant and treated with crosslinking reagents revealed no shift in mobility of the mutant protein (data not shown), suggesting that Cys388 (TM7) and Cys433 (TM8) failed to cross-link, indicating that the spatial orientation of Cys388 and R433C residues may prevent cross-linking from occurring.

Because we failed to observe cross-linking between R433C (TM8) and Cys388 (TM7), we introduced point mutations along TM8. Fifteen cysteine single-point mutations were introduced along this transmembrane region, between amino acids 431 and 446, with the goal of elucidating whether TMs 7 and 8 were situated in close proximity. Each mutant contains four cysteines, three endogenous and one introduced.
Mutant proteins were expressed, and their correct trafficking was verified by confocal microscopy. We characterized the plasma membrane vesicles by immunoblotting and observed that all the mutant proteins were expressed to a similar degree.

We next investigated the possibility of cross-linking the inserted cysteines with endogenous Cys388 using cross-linking reagents of varying lengths (M5M, M8M, M17M, CuPhen). None of the reagents were able to cross-link the mutants.

Cross-linking analysis of proteins with a mutation in TM8 did not reveal the existence of any cysteine pairs with Cys388 (TM7), despite the fact that these cysteines are predicted to be close enough for such cross-linking to occur on the basis of the bMRP1 structures.

Competition between Substrates and a Cross-Linker in R433C/E1144C and I1193C Mutants of 3Cys $\Delta$ MRP1. To assess the possibility that the cross-linking observed between mutants I1193C and $\mathrm{R} 433 \mathrm{C} / \mathrm{E} 1144 \mathrm{C}$ may have been the result of mutationally induced changes in the protein tertiary structure, we compared $\mathrm{LTC}_{4}$ uptake by these mutants with that of $3 \mathrm{Cys} \Delta \mathrm{MRP} 1$. The plasma membrane vesicles of both mutants showed ATP-dependent MRP1-mediated uptake of ${ }^{3} \mathrm{H}$ labeled $\mathrm{LTC}_{4}$. We normalized the amount of MRP1 through densitometry analysis of the immunoblots to compare transport activity between these proteins. As summarized in Supplemental Table 2, the level of $\mathrm{LTC}_{4}$ uptake for both mutants was comparable to that of 3 Cys $\Delta \mathrm{MRP} 1$. This suggests that tertiary structure of MRP1 was not altered by introduction of cysteine residues.

Mutational evidence exists that TM8, where the mutation R433C was introduced, forms part of the drug-binding pocket of MRP1 (Grant et al., 2008). Consequently, we examined the effect of substrate binding on cross-linking of the mutant R433C/E1144C 3Cys $\Delta$ MRP1. Plasma membrane vesicles were preincubated with MRP1 substrates, $\mathrm{LTC}_{4}$ or estrone sulfate, in the presence of $S$-methyl-GSH, at $23^{\circ} \mathrm{C}$ for 15 minutes. $S$-methyl-GSH, rather than GSH (Qian et al., 2001), was chosen to stimulate estrone sulfate binding to avoid a possible GSH reaction with thiosulfonates. After preincubation of the membranes with substrate, the samples were cooled on ice and treated with the cross-linking reagent M8M for 15 minutes, followed by immunoblot analysis. Cross-linking was observed in all experiments, independent of the substrate employed. 
The degree of cross-linking was comparable for all substrate concentrations tested. Our data suggested that neither $\mathrm{LTC}_{4}$ nor estrone sulfate influenced the cross-linking reactions of R433C/E1144C 3Cys $\Delta \mathrm{MRP} 1$.

We also tested the effect of the same substrates (i.e., LTC $_{4}$ and estrone sulfate) on the cross-linking of another mutant, I1193C 3Cys $\Delta$ MRP1, located in TM16. The introduced cysteine residue is situated close to Y1189 and Y1190, both of which have been reported to aid in the transport of MRP1 substrates. The substitution of these residues with Ala or Ser reduced the transport activity of MRP1 (Conseil et al., 2005). The most dramatic effect was observed on GSH transport, which was reduced 10-30\% compared with wild-type MRP1 (Conseil et al., 2005). These observations suggested that I1193C might also be located in the drug-binding pocket. Consequently, we examined whether cross-linking involving I1193C was inhibited in the presence of substrate.

Membrane vesicles of I1193C 3Cys $\Delta$ MRP1 mutant were treated and analyzed as described above. Immunoblotting revealed that the intensity of the X-link product was decreased in a concentration-dependent manner by estrone sulfate but not by LTC $_{4}$. As shown in Fig. 5 and Supplemental Fig. 3, estrone sulfate can eliminate cross-linking of I1193C 3Cys $\Delta \mathrm{MRP} 1$ at concentration above $2.5 \mu \mathrm{M}$. Estrone sulfate treatment was performed in presence of fixed concentration of $S$-methyl GSH, which suggests that estrone sulfate rather than $S$-methyl GSH is the active compound in inhibition of the cross-linking reaction. These data suggest that cross-linking occurs within the estrone sulfate binding pocket of MRP1, in the space between TMs 7 and 16 .

Residues C388 (TM7), R433C (TM8), E1144C (TM15), and I1193C (TM16) Are Accessible to the Extracellular Environment. It is unknown whether the translocation pore in MRP1 is accessible to the extracellular aqueous medium in the closed conformation. Many MRP1 substrates are organic anions, which are predominantly charged, hydrophilic molecules. Although hydrophobic compounds can be transported by MRP1, their transport often requires the presence of a hydrophilic GSH molecule (Rothnie et al., 2008). This strongly suggests that when the protein is in a high-affinity state, the substrate and/or GSHbinding site(s) must be accessible from the cytosol and that the translocation pore is probably closed to the extracellular space. Conversely, when in a low-affinity state, the reverse might be expected to occur. We performed a series of studies to test this hypothesis and to identify which residues in the predicted translocation pathway are accessible to the aqueous phase from the extracellular space.

Two thiol-charged compounds, MTSES and MTSEA, have proven to be useful probes in evaluating the aqueous accessibility of cysteine residues in channels and transporters (Karlin and Akabas, 1998). MTSEA, a membrane-permeable reagent, can penetrate the membrane bilayer and covalently bind to the cysteines of a wide range of proteins. In contrast, MTSES is a membrane-impermeable reagent that, in intact cells, reacts only with cysteines accessible from the extracellular space. If the translocation pore of MRP1 is accessible from the extracellular space, theoretically both MTSEA and MTSES may potentially inhibit reagent-mediated cross-linking of cysteine pairs on the cytosolic side of the membrane (Loo et al., 2004).

We first examined the R433C/E1144C mutant of 3Cys $\Delta \mathrm{MRP} 1$ to determine whether these cysteines are accessible from the extracellular matrix by both reagents. Intact cells transiently expressing the R433C/E1144C mutant were treated with MTSEA, MTSES, or with neither reagent. After extensive washing with buffer, cells were harvested and frozen. Plasma membrane vesicles were prepared and then treated with M5M and M8M. The cross-linking reactions were performed in a hypotonic phosphate buffer that has been shown to transform the vesicles into membrane sheets (Rothnie et al., 2006) such that all unmodified cysteines would be accessible to thiosulfonate crosslinking reagents.

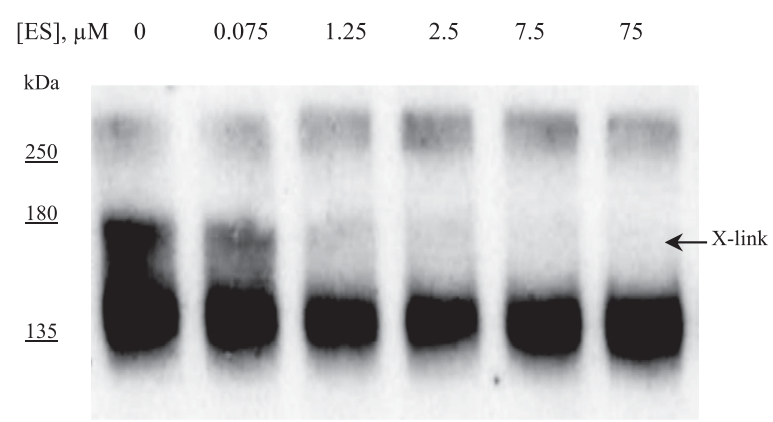

Fig. 5. Competition of estrone sulfate with cross-linker in I1193C 3Cys $\Delta$ MRP1. I1193C mutant membrane sheets were preincubated with various concentrations of estrone sulfate $(0-75 \mu \mathrm{M}), 1 \mathrm{mM} \mathrm{S}$-methyl-GSH, and $10 \mathrm{mM} \mathrm{MgCl}_{2}$ for 15 minutes at $23^{\circ} \mathrm{C}$. The samples were then cooled to $4^{\circ} \mathrm{C}$ and treated with $50 \mu \mathrm{M}$ M8M. The mixtures were subjected to immunoblot analysis with anti-His tag antibody. Densitometric analysis of the immunoblot can be found at Supplemental Fig. SI 3).

Immunoblot analysis of the MTSEA- and MTSES-treated membranes is shown in Fig. 6A. In the case of membrane-permeable MTSEAtreated membranes, an absence of the cross-linked species indicated that the cysteine pair was accessible and modified by the MTSEA reagent. The membrane-impermeable MTSES-treated membranes, on the other hand, displayed three bands (Fig. 6A). According to densitometry analysis of the immunoblots, $30 \%$ of MRP1 remained unmodified $(150 \mathrm{kDa}), 50 \%$ underwent intermolecular cross-linking (350 kDa)most likely involving cysteines in NDB2-and 20\% appeared as an intramolecular cross-link product (X-link; $220 \mathrm{kDa}$ ). These results suggested that cross-linking between Cys433 and Cys1144 was inhibited to some extent by the presence of MTSES, which, in turn, implied that Cys433 (TM8) and/or Cys1144 (TM15) were partially accessible to the external aqueous medium.

The same approach was used to study the second available mutant, I1193C 3Cys $\Delta$ MRP1, where the cysteine residues (C388 and C1193) are located in TMs 7 and 16, respectively. MTSEA completely inhibited cross-linking reactions, and MTSES partially inhibited the formation of cross-links (Fig. 6B). Likewise, these results indicate that Cys388 and/or the amino acid residue at position 1193 is/are partially accessible from the extracellular side of a membrane.

The cross-linking experiments described above were performed using intact cells in the presence of either MTSEA or MTSES reagents, which were then further cross-linked when in the form of membrane sheets. However, the distribution of MRP1 in intact cells is quite complex. Although the majority of MRP1 translocates to the plasma membrane, some of the protein is expressed in different cellular compartments, especially in endocytotic vesicles (Almquist et al., 1995). MRP1 that is not expressed in the plasma membrane would be inaccessible to the membrane-impermeable MTSES reagent. Consequently, its cysteines would remain capable of cross-linking, which might explain the observed residual cross-linking of MRP1 in MTSES-treated cells. Another possible reason for residual cross-linking of MRP1 in MTSES-treated cells could involve the MRP1 conformation. With MRP1 in intact cells, where exposure to ATP and glutathione cannot be readily controlled, the protein is expected to exist in both the open (low affinity) and closed (high affinity) conformations.

To address these issues, we used membrane vesicles instead of intact cells. Plasma membrane vesicles of mutants R433C/E1144C (TMs 8 and $15)$ were treated with MTSEA and MTSES reagents. The reactions were terminated by quick removal of the reagents through gel-filtration columns. Membrane vesicles were then transformed into membrane sheets, as described in Materials and Methods, and probed for crosslinking under standard conditions (50 $\mu \mathrm{M}$ cross-linking; 15 minutes on 

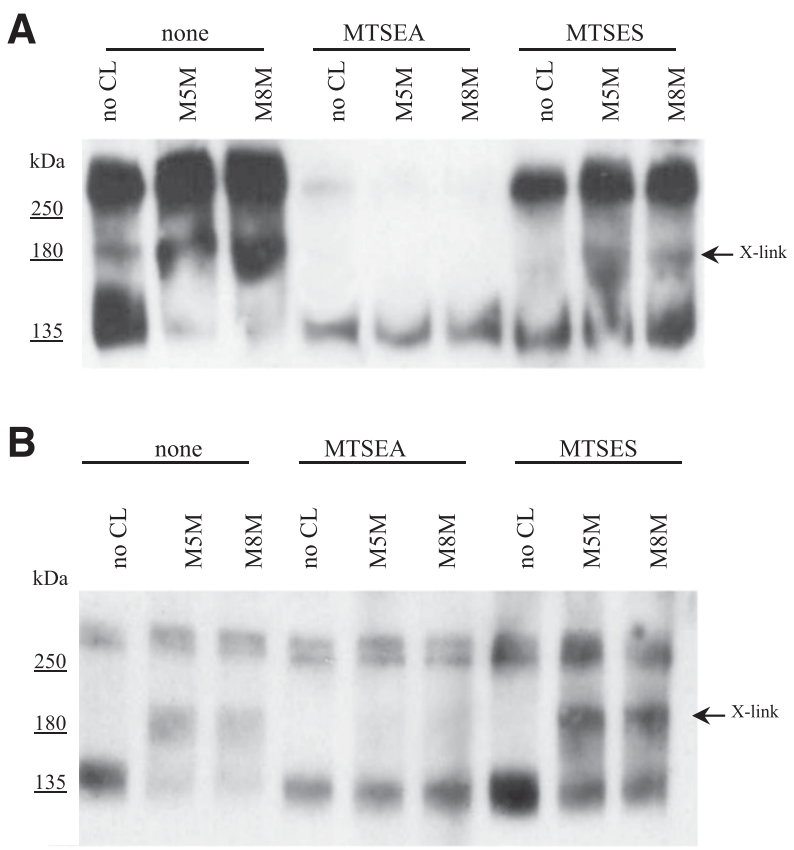

Fig. 6. Effect of MTSEA and MTSES on cross-linking in whole cells expressing 3Cys $\triangle$ MRP1 mutants R433C/E1144C (A) and I1193C (B). Cells were treated with $10 \mathrm{mM}$ MTSES, $2.5 \mathrm{mM}$ MTSEA, or buffer (none) for 15 minutes at $23^{\circ} \mathrm{C}$. The treatment was stopped by dilution with ice-cold PBS and cells were washed with PBS to remove the reagents. Membrane sheets were prepared and treated with $50 \mu \mathrm{M}$ M5M or M8M, or without the cross-linker (no CL) for 15 minutes on ice. Protein mixtures were run on $7.5 \%$ SDS-PAGE gel, followed by immunoblot analysis with anti-His tag antibody. The position of the cross-linked product is indicated (X-link).

ice). Immunoblot analysis of the MTSEA/MTSES-treated membranes revealed the absence of cross-linking events, whereas untreated membranes were effectively cross-linked (Fig. 7A). On the basis of this observation, we concluded that Cys1144 (TM15) and Cys433 (TM8), under ATP-free conditions, were modified by MTSES.

Finally, we probed membrane vesicles of the I1193C 3Cys $\Delta$ MRP1 mutant, containing two cysteines located in TMs 7 and 16, with membrane-permeable and -impermeable MTS reagents. MTSEA and MTSES blocked cross-linking reactions induced by thiosulfonate reagents (Fig. 7B). These results suggested that MTSES has access to Cys388 and/or the amino acid at position 1193 (TM16).

\section{Discussion}

Cysteine-scanning mutagenesis coupled with cross-linking studies provides a powerful tool for investigating structure and dynamics of transmembrane proteins in the native environment of a cell membrane (Stauffer and Karlin, 1994). Successful cysteine-scanning mutagenesis requires a fully functional protein, ideally devoid of all cysteines. However, full-length MRP1 presented a challenge to this technique because the protein contains 25 cysteines. We have previously described the construction of "almost" cysless $\triangle \mathrm{MRP1}$ (3Cys $\Delta$ MRP1) that retains the ability to traffic to the PM and to transport several MRP1 substrates at a normal rate (Qin et al., 2012). In this study, we first examined the feasibility of using $3 \mathrm{Cys} \Delta \mathrm{MRP} 1$ as a template for cysteine-scanning mutagenesis and cysteine crosslinking studies. We found no evidence of intramolecular disulfide cross-linking involving any of the remaining three endogenous cysteines in the 3Cys $\triangle$ MRP1 template. Furthermore, all 25 mutants in which additional cysteines had been introduced into the 3Cys $\Delta$ MRP1 template remained functional and trafficked to the PM.
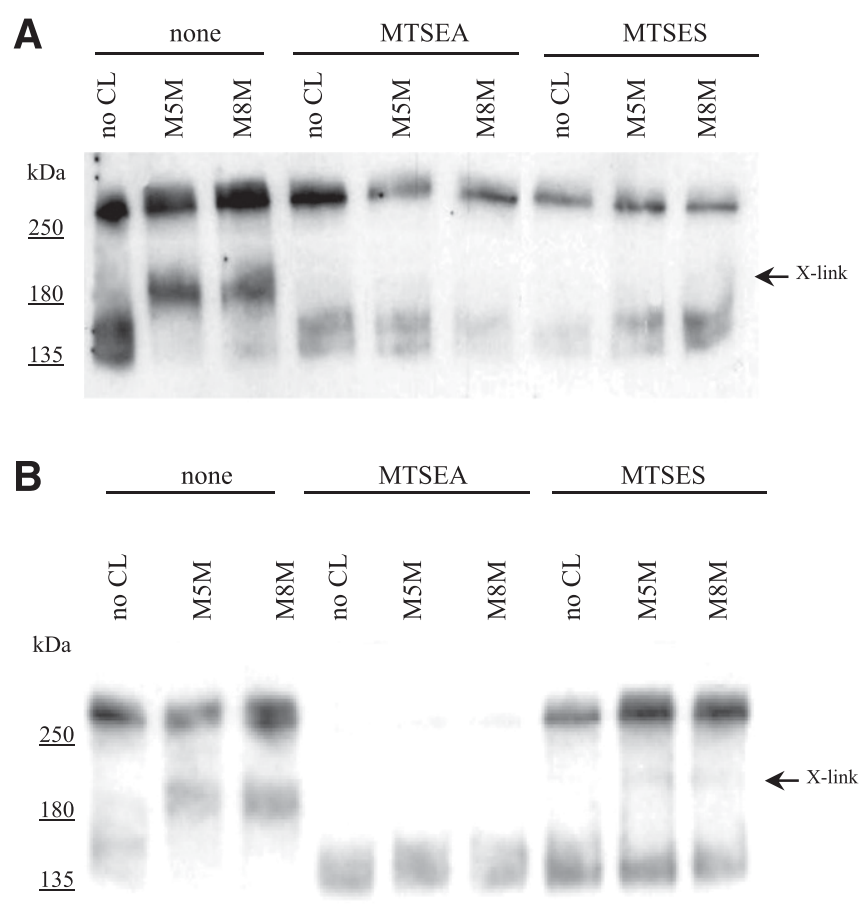

Fig. 7. Effect of MTSEA and MTSES on cross-linking of 3 Cys $\triangle M R P 1$ mutants. Membranes prepared from HEK293 cells expressing mutants R433C/E1144C (A) and $\mathrm{I} 1193 \mathrm{C}$ (B) were incubated at $23^{\circ} \mathrm{C}$ for 15 minutes in the presence of $10 \mathrm{mM}$ MTSES or $2.5 \mathrm{mM}$ MTSEA, or without either reagent (none). MTSEA or MTSES reagents were removed from membranes using G-50 spin columns. Plasma membrane-rich flow-through fractions were then treated with $50 \mu \mathrm{M}$ M5M or M8M, or without the cross-linker (no CL) for 15 minutes on ice. The reactions were mixed with SDS loading buffer containing EDTA and no reducing agent, and then run on $7.5 \%$ SDS-PAGE gel, followed by immunoblot analysis with anti-His tag antibody. The position of the cross-linked products is indicated (X-link).

Overall, the results of these studies strongly support the potential utility of 3 Cys $\triangle \mathrm{MRP} 1$ as a template for cysteine-scanning mutagenesis.

The spatial relationships among the transmembrane helices were investigated by disulfide cross-linking experiments using mutants of the 3Cys $\triangle \mathrm{MRP} 1$ and cross-linkers of differing lengths. Cross-linking was observed between cysteine pairs in TM7/TM16, TM7/TM15, TM8/TM15, and TM8/TM14 (Table 1). Based on the lengths of the cross-linking agents, our results indicated that TM7 was 6-9 and 14$24 \AA$ apart from TMs 16 and 15, respectively, and that TM8 was 6-9 and 13-24 Å apart from TMs 15 and 14 (Fig. 8A). These distances are in reasonable agreement with the hMRP1 model and recently obtained bMRP1 structures (Johnson and Chen, 2017).

In contrast, we found considerable discrepancies between distances predicted by both the hMRP1 model and the cryo-EM structure of bMRP1 and those suggested by our cross-linking studies with the R433C/E1144C (TMs 8 and 15) mutant. The distance between these residues in the hMRP1 model and the cryo-EM structure of bMRP1 is predicted to be $17.1 \AA$. However, we found that the two cysteine residues in the mutant protein could be cross-linked by M5M, indicating that they are no more than $9 \AA$ apart. The fact that $433 \mathrm{C}$ can be cross-linked to $1144 \mathrm{C}$ suggests that the side chain of amino acid at position 433 is projecting toward side chain of amino acid position 1144, and thus toward the translocation pore. Neither the bMRP1 structure nor the hMRP1 model supports this finding. In both predicted structures, R433 projects away from the translocation pore. However, in bMRP1 (apo and $\mathrm{LTC}_{4}$-bound structures) the adjacent residue, $\mathrm{Q} 432$, does project toward 
the translocation pore and the distance between the $\alpha$-carbon atoms of Q432 and E1143 in bMRP1 (E1143 occupies the equivalent position to E1144 in hMPR1) is predicted to be $12.4 \AA$, which is nearer to, but still longer than, what was detected experimentally by cross-linking. This finding suggests that there may be some structural differences between native MRP1 in the lipid bilayer and the models derived from the crystallographic structure of Sav1866 and the cryo-EM structures of bMRP.

Under physiologic conditions it is possible that MRP1 is not static and that transmembrane helices are flexible and dynamic (Tanizaki and Feig, 2006). Thus the experimental conditions under which cross-linking was performed may allow cross-linking of only a subset of potential states of MRP1, and we cannot exclude existence of other possible states of the protein that fail to cross-link. To minimize thermal motion of the transmembrane domains, we performed cross-linking experiments at the lowest possible temperature. Nevertheless, we cannot exclude the possibility that remaining thermal motion may bring two transmembrane helices close enough for cross-linking to occur in a subset of conformations that do not necessarily represent the predominant state of the protein. This might be an explanation for the discrepancy in distances between our results and the cryo-EM structure of bMRP1. On the other hand, the lack of cross-linking provides a strong indication that the distance between the cysteine pairs exceeds the length of a crosslinker under any conditions.

Another instance of inconsistency between the bMRP1 structure, the hMRP1 model, and our cross-linking results occurred with mutants I1193C and Y1190C (TM16). I1193C, but not Y1190C, cross-linked to Cys388 (TM7). According to the hMRP1 model, the Y1190 residue is predicted to project directly toward Cys388, whereas the side chain of I1193 projects away from Cys388 and toward the translocation pore. Thus, the cross-linking results are the reverse of what would be anticipated. The bMRP1 structure depicts Cys388 situated on the side of TM7 facing TM8 and projecting away from the translocation pore. This orientation was not supported by our cross-linking results. Considering that MRP1 in a living cell is usually exposed to a high level of ATP and GSH (one of its substrates/modulators) and embedded in a lipid bilayer, one might expect that both the hMRP1 model (ADPvanadate-trapped) and the bMRP1 structures (in the absence and presence of $\mathrm{LTC}_{4}$, no nucleotide) may not totally represent the state of the protein in its natural environment. Therefore, our 3Cys $\triangle \mathrm{MRP1}$ template may provide a useful comparative tool for probing the conformations of MRP1 in its native environment.

In addition, we attempted to probe the substrate-binding site(s) within 3Cys $\triangle$ MRP1 cysteine mutants by investigating the ability of well characterized MRP1 substrates to inhibit cross-linking. One such substrate, estrone sulfate, effectively abrogated cross-linking of Cys388 (TM7) and I1 193C (TM16), which suggests that estrone sulfate may interact with residues in TM7 and/or TM16 that are close to the cytosolic interface of the translocation pore. This finding agrees with previous mutagenesis experiments in which the mutation of residues 1189 or 1190 in TM16 led to reduced transport of several MRP1 substrates (Conseil et al., 2005). However, the high-affinity substrate, $\mathrm{LTC}_{4}$, failed to inhibit cross-linking of these cysteines (Cys388 and I1193C). The variable effects of these two substrates are consistent with the hypothesis that MRP1 contains multiple and somewhat overlapping binding domains within a larger, flexible pocket (Deeley et al., 2006).

Using the same strategy (competition between a substrate and a crosslinker), we probed another area of the predicted translocation pore situated between R433C (TM8) and E1144C (TM15). In this case, we were unable to demonstrate substrate-dependent inhibition of crosslinking by any substrates tested, despite the fact that mutation of these residues reduces estrone sulfate transport in the case of E1144A (Conseil

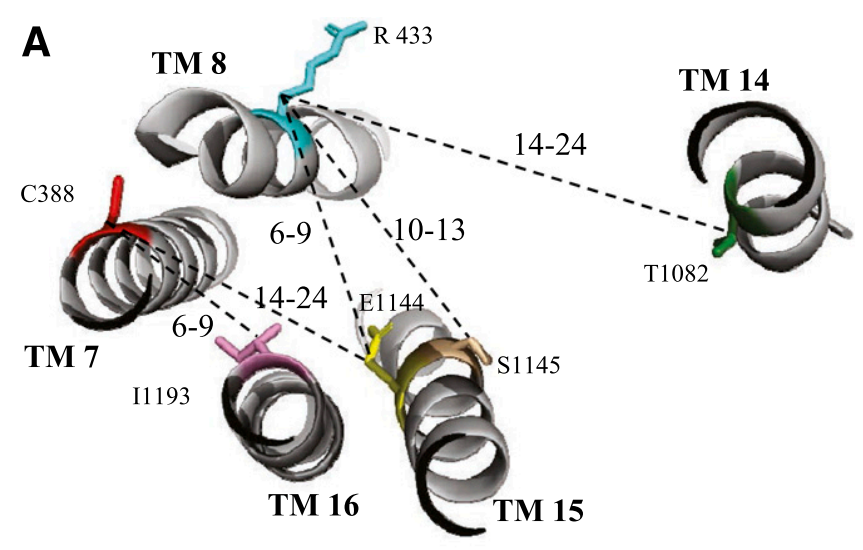

B

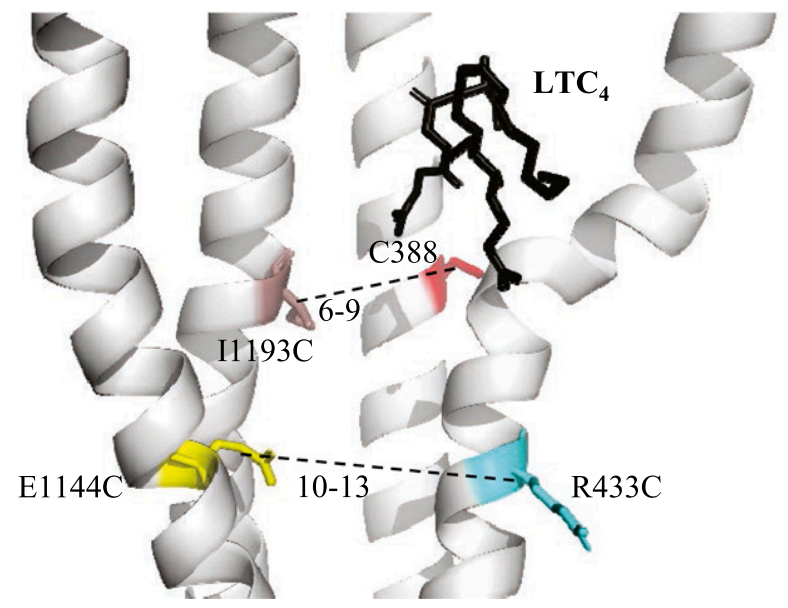

Fig. 8. Structural features of MRP1. (A) Determining the dimensions of the hMRP1 translocation pore employing thiol cross-linking reagents. Shown is the arrangement of TM helices (on the basis of bMRP1 PDB ID: 5uj9) as viewed from the extracellular face of the membrane. Numbers represent distances (in angstroms) deduced by results of cross-linking experiments using varied-length cross-linking reagents. (B) Spatial arrangement of cysteine pairs (C388/I1193C and R433C/E1144C) in bMRP1 structure in presence of $\mathrm{LTC}_{4}$ (PDB ID: 5uja). Shown is the view from the plane perpendicular to the lipid bilayer. Dotted lines represent a cross-link in each mutant.

et al., 2009) and decreases $\mathrm{LTC}_{4}$ transport in the case of R433S (Conrad et al., 2002). Overall, the results suggest that these residues may not interact directly with the tested substrates but could be located within or near the substrate-binding pocket. The recent cryo-EM structure of bMRP1 in the presence of $\mathrm{LTC}_{4}$ (Johnson and Chen, 2017) may provide an explanation for the different response of two tested mutants, i.e., the lack of inhibition of cross-linking by any of the substrates tested in the case of R433C/E1144C mutant and selective inhibition in the case of C388/I1193C mutant. As can be seen in the bMRP1 structure (Fig. 8B), E1144 and R433 are located quite far from the $\mathrm{LTC}_{4}$ binding pocket, whereas C388 and I1 193 are closer. It might be inferred that the presence of $\mathrm{LTC}_{4}$ would not interfere with cross-linking of R433C/E1144C pair but might inhibit C388/I1 193C cross-linking. Although we observed no inhibition of the cross-linking of C388 and $\mathrm{I}_{1193 C}$ by $\mathrm{LTC}_{4}$, crosslinking was strongly inhibited by estrone sulfate. This may be attributable to the binding of estrone sulfate within the same pocket but in an orientation such that it binds closer to the C388/I1193C residues. This result supports the previously postulated idea of a GSH-bindinginduced conformational change that can unmask the estrone sulfate binding site (Rothnie et al., 2008). 
Finally, we used the C388/I1193C and E1144C/R433C mutants to investigate the aqueous accessibility of the binding pocket as a whole. We hypothesized that when MRP1 is expressed in cell membranes, the substrate and/or GSH-binding site(s) must be accessible from the cytosol, and that the translocation pore is presumably closed to the extracellular space. However, our experiments with whole cells expressing R433C/E1144C or I1193C mutants, as well as with membrane vesicles (representing an ATPfree environment), revealed that residues at positions 388 (TM7), 433 (TM8), 1144 (TM15), and 1193 (TM16) are accessible to the extracellular aqueous medium. Interestingly, similar results were described for P-gp (Loo et al., 2004), also indicating that the drugbinding site of P-gp is accessible from the aqueous milieu on the extracellular side of a membrane, despite the dissimilar nature of the substrates for P-gp and MRP1. Most the P-gp substrates are believed to diffuse freely through the cell membrane, whereas many MRP1 substrates are relatively hydrophilic, and the transport of some substrates (both hydrophobic and hydrophilic) depends on or is stimulated by hydrophilic GSH molecules.

In summary, our results support the suitability of "almost" cysless $\Delta \mathrm{MRP} 1$ as a template for cysteine-scanning mutagenesis coupled with cross-linking studies. Use of this template has provided and may continue to provide significant insight into the complex role of MRP1 in the binding and transport of various drugs and chemicals.

\section{Acknowledgments}

The authors thank Monika Vasa for excellent technical assistance and Matthew Gordon for microscopy assistance. We also thank Dr. Gwenaëlle Conseil for fruitful discussions and Anthony Zara for manuscript preparation.

\section{Authorship Contributions}

Participated in research design: Trofimova, Deeley.

Conducted experiments: Trofimova.

Performed data analysis: Trofimova, Deeley.

Wrote or contributed to the writing of the manuscript: Trofimova, Deeley.

\section{References}

Aller SG, Yu J, Ward A, Weng Y, Chittaboina S, Zhuo R, Harrell PM, Trinh YT, Zhang Q, Urbatsch IL, et al. (2009) Structure of P-glycoprotein reveals a molecular basis for poly-specific drug binding. Science 323:1718-1722.

Almquist KC, Loe DW, Hipfner DR, Mackie JE, Cole SP, and Deeley RG (1995) Characterization of the M(r) 190,000 multidrug resistance protein (MRP) in drug-selected and transfected human tumor cell. Cancer Res 55:102-110.

Bakos E, Hegedüs T, Holló Z, Welker E, Tusnády GE, Zaman GJ, Flens MJ, Váradi A, and Sarkadi B (1996) Membrane topology and glycosylation of the human multidrug resistance-associated protein. J Biol Chem 271:12322-12326.

Bakos E and Homolya L (2007) Portrait of multifaceted transporter, the multidrug resistanceassociated protein 1 (MRP1/ABCC1). Pflugers Arch 453:621-641.

Chan HS, Lu Y, Grogan TM, Haddad G, Hipfner DR, Cole SP, Deeley RG, Ling V, and Gallie BL (1997) Multidrug resistance protein (MRP) expression in retinoblastoma correlates with the rare failure of chemotherapy despite cyclosporine for reversal of P-glycoprotein. Cancer Res 57: 2325-2330.

Cole SP (2014) Multidrug resistance protein 1 (MRP1, ABCC1), a "multitasking" ATP-binding cassette (ABC) transporter. J Biol Chem 289:30880-30888.

Cole SP, Bhardwaj G, Gerlach JH, Mackie JE, Grant CE, Almquist KC, Stewart AJ, Kurz EU, Duncan AM, and Deeley RG (1992) Overexpression of a transporter gene in a multidrugresistant human lung cancer cell line. Science 258:1650-1654.
Conrad S, Kauffmann HM, Ito K, Leslie EM, Deeley RG, Schrenk D, and Cole SP (2002) A naturally occurring mutation in MRP1 results in a selective decrease in organic anion transpor and in increased doxorubicin resistance. Pharmacogenetics 12:321-330.

Conseil G, Deeley RG, and Cole SP (2005) Role of two adjacent cytoplasmic tyrosine residues in MRP1 (ABCC1) transport activity and sensitivity to sulfonylureas. Biochem Pharmacol 69:451-461.

Conseil G, Rothnie AJ, Deeley RG, and Cole SP (2009) Multiple roles of charged amino acids in cytoplasmic loop 7 for expression and function of the multidrug and organic anion transporter MRP1 (ABCC1). Mol Pharmacol 75:397-406.

Dawson RJ and Locher KP (2006) Structure of a bacterial multidrug ABC transporter. Nature 443: 180-185.

Deeley RG and Cole SP (2006) Substrate recognition and transport by multidrug resistance protein 1 (ABCC1). FEBS Lett 580:1103-1111.

Deeley RG, Westlake C, and Cole SP (2006) Transmembrane transport of endo- and xenobiotics by mammalian ATP-binding cassette multidrug resistance proteins. Physiol Rev 86:849-899.

DeGorter MK, Conseil G, Deeley RG, Campbell RL, and Cole SP (2008) Molecular modeling of the human multidrug resistance protein 1 (MRP1/ABCC1). Biochem Biophys Res Commun 365: 29-34.

Grant CE, Gao M, DeGorter MK, Cole SP, and Deeley RG (2008) Structural determinants of substrate specificity differences between human multidrug resistance protein (MRP) 1 (ABCC1) and MRP3 (ABCC3). Drug Metab Dispos 36:2571-2581.

Hipfner DR, Almquist KC, Leslie EM, Gerlach JH, Grant CE, Deeley RG, and Cole SP (1997) Membrane topology of the multidrug resistance protein (MRP). A study of glycosylation-site mutants reveals an extracytosolic NH2 terminus. J Biol Chem 272:23623-23630.

Hipfner DR, Deeley RG, and Cole SP (1999) Structural, mechanistic and clinical aspects of MRP1. Biochim Biophys Acta 1461:359-376.

Johnson ZL and Chen J (2017) Structural basis of substrate recognition by the multidrug resistance protein MRP1. Cell 168:1075-1085.e9.

Karlin A and Akabas MH (1998) Substituted-cysteine accessibility method. Methods Enzymol 293: 123-145.

Leslie EM, Deeley RG, and Cole SP (2005) Multidrug resistance proteins: role of P-glycoprotein, MRP1, MRP2, and BCRP (ABCG2) in tissue defense. Toxicol Appl Pharmacol 204:216-237. Loe DW, Almquist KC, Deeley RG, and Cole SP (1996) Multidrug resistance protein (MRP)mediated transport of leukotriene $\mathrm{C} 4$ and chemotherapeutic agents in membrane vesicles. Demonstration of glutathione-dependent vincristine transport. J Biol Chem 271:9675-9682

Loo TW, Bartlett MC, and Clarke DM (2004) The drug-binding pocket of the human multidrug resistance P-glycoprotein is accessible to the aqueous medium. Biochemistry 43:12081-12089.

Loo TW and Clarke DM (1996) Inhibition of oxidative cross-linking between engineered cysteine residues at positions 332 in predicted transmembrane segments (TM) 6 and 975 in predicted TM12 of human P-glycoprotein by drug substrates. J Biol Chem 271:27482-27487.

Qian YM, Song WC, Cui H, Cole SP, and Deeley RG (2001) Glutathione stimulates sulfated estrogen transport by multidrug resistance protein 1. J Biol Chem 276:6404-6411.

Qin L, Tam SP, and Deeley RG (2012) Effect of multiple cysteine substitutions on the functionality of human multidrug resistance protein 1 expressed in human embryonic kidney 293 cells: identification of residues essential for function. Drug Metab Dispos 40:1403-1413.

Rothnie A, Callaghan R, Deeley RG, and Cole SP (2006) Role of GSH in estrone sulfate binding and translocation by the multidrug resistance protein 1 (MRP1/ABCC1). J Biol Chem 281: 13906-13914.

Rothnie A, Conseil G, Lau AY, Deeley RG, and Cole SP (2008) Mechanistic differences between GSH transport by multidrug resistance protein 1 (MRP1/ABCC1) and GSH modulation of MRP1-mediated transport. Mol Pharmacol 74:1630-1640.

Slot AJ, Molinski SV, and Cole SP (2011) Mammalian multidrug-resistance proteins (MRPs). Essays Biochem 50:179-207.

Stauffer DA and Karlin A (1994) Electrostatic potential of the acetylcholine binding sites in the nicotinic receptor probed by reactions of binding-site cysteines with charged methanethiosulfonates. Biochemistry 33:6840-6849.

Taguchi Y, Yoshida A, Takada Y, Komano T, and Ueda K (1997) Anti-cancer drugs and glutathione stimulate vanadate-induced trapping of nucleotide in multidrug resistance-associated protein (MRP). FEBS Lett 401:11-14.

Tanizaki S and Feig M (2006) Molecular dynamics simulations of large integral membrane proteins with an implicit membrane model. J Phys Chem B 110:548-556.

Westlake CJ, Cole SP, and Deeley RG (2005) Role of the NH2-terminal membrane spanning domain of multidrug resistance protein $1 / \mathrm{ABCC} 1$ in protein processing and trafficking. Mol Biol Cell 16:2483-2492.

Zhang DW, Gu HM, Situ D, Haimeur A, Cole SP, and Deeley RG (2003) Functional importance of polar and charged amino acid residues in transmembrane helix 14 of multidrug resistance protein 1 (MRP1/ABCC1): identification of an aspartate residue critical for conversion from a high to low affinity substrate binding state. J Biol Chem 278:46052-46063.

Address correspondence to: Dr. Roger G. Deeley, Division of Cancer Biology and Genetics, Rm. 300, 10 Stuart St., Queen's University Cancer Research Institute, Kingston, ON K7L 3N6, Canada. E-mail: deeleyr@queensu.ca 